\title{
Inhibition of CPT1a as a Prognostic Marker can Synergistically Enhance the Antileukemic Activity of ABT199
}

\section{Shihui Mao}

Zhejiang University School of Medicine First Affiliated Hospital https://orcid.org/0000-0001-6282-4652

\section{Qing Ling}

Zhejiang University School of Medicine First Affiliated Hospital

\section{Jiajia Pan}

First Hospital of Zhejiang Province: Zhejiang University School of Medicine First Affiliated Hospital

\section{Fenglin Li}

First Hospital of Zhejiang Province: Zhejiang University School of Medicine First Affiliated Hospital

\section{Shujuan Huang}

First Affiliated Hospital of Anhui Medical University

\section{Wenle Ye}

First Hospital of Zhejiang Province: Zhejiang University School of Medicine First Affiliated Hospital

\section{Wenwen Wei}

First Hospital of Zhejiang Province: Zhejiang University School of Medicine First Affiliated Hospital

\section{Xiangjie Lin}

First Hospital of Zhejiang Province: Zhejiang University School of Medicine First Affiliated Hospital

\section{Yu Qian}

First Hospital of Zhejiang Province: Zhejiang University School of Medicine First Affiliated Hospital

\section{Yungui Wang}

First Hospital of Zhejiang Province: Zhejiang University School of Medicine First Affiliated Hospital

\section{Xin Huang}

First Hospital of Zhejiang Province: Zhejiang University School of Medicine First Affiliated Hospital

Jiansong Huang

First Hospital of Zhejiang Province: Zhejiang University School of Medicine First Affiliated Hospital Jinghan Wang

First Hospital of Zhejiang Province: Zhejiang University School of Medicine First Affiliated Hospital Jie Jin ( $\nabla_{\text {jiej0503@zju.edu.cn ) }}$

The First Affiliated Hospital, Zhejiang University College of Medicine https://orcid.org/0000-0002-81669915 
Keywords: ABT199, Carnitine palmitoyltransferase 1, Fatty acid oxidation, oncogene, Acute myeloid leukemia

Posted Date: February 26th, 2021

DOI: https://doi.org/10.21203/rs.3.rs-255509/v1

License: (c) (1) This work is licensed under a Creative Commons Attribution 4.0 International License. Read Full License 


\section{Abstract}

Background: Fatty acid oxidation (FAO) provides an important source of energy to promote the growth of leukemia cells. Carnitine palmitoyltransferase $1 \mathrm{a}(\mathrm{CPT} 1 \mathrm{a})$, a rate-limiting enzyme of the essential step of FAO, can facilitate cancer metabolic adaptation. Previous reports demonstrated that CPT1a acts as a potential molecular target in solid tumors and hematologic disease. However, no systematic study was conducted to explore the its prognostic value and possible treatment strategies on acute myeloid leukemia (AML).

Methods: The expression of CPT1a in 325 cytogenetically normal AML (CN-AML) cases was evaluated using RT-PCR. The combination effects of ST1326 and ABT199 were studied in AML cells and primary patients. MTS was used to measure the cell proliferation rate. Annexin V/propidium iodide staining and flow cytometry analysis was used to measure the apoptosis rate. western blot were used to measure the expression of $\mathrm{Mcl}-1$.

Results: In this study, we found AML patients with high CPT1a expression $(n=245)$ had a relatively short overall survival $(P=0.01)$ and event free survival $(P=0.032)$ compared to patients in low expression group $(n=80)$. In parallel, downregulation of CPT1a inhibits proliferation of AML cells. We also performed a metabolomic analysis and observed a decrease of fatty acid in CPT1a high expression group comparing to low expression group, which confirms that CPT1a can facilitate FAO to provide energy fueling tumor growth. Moreover, we found downregulation of CPT1a sentitizes BCL-2 inhibitor ABT199 and CPT1aselective inhibitor ST1326 combined with ABT199 has a strong synergistic effect to induce apoptosis in AML cells and primary patient blasts for the first time. The underlying synergistic mechanism might be that ST1326 inhibits pGSK3 $\beta$ and pERK expression, leading to downregulation of Mcl-1.

Conclusion: our study indicates that overexpression of CPT1a predicts poor clinical outcome in AML. CPT1a-selective inhibitor ST1326 combined with Bcl-2 inhibitor ABT199 showed strongsynergistic inhibitory effects on AML.

\section{Introduction}

Acute myeloid leukemia (AML) is a group of the heterogeneous disease characterized by the clonal proliferation of immature myeloid cells(1). For young patients, a combination of cytarabine and anthracycline has been used as the standard induction regimen over the last four decades $(2,3)$. While, recently some novel drugs like Bcl-2 inhibitor are proved to be effective for old AML patients. Although the advances in the new drugs for AML treatment are impressive, the 5-year overall survival rate is frustratingly low for adults (25\%) andelderly patients(10\%) (4). Thus, research on novel drugs and rational combination therapies is imperative.

Fatty acid oxidation (FAO) is an important source of NADH, FADH2, NADPH and ATP fueling tumor growth in conditions of metabolic stress(5). Carnitine palmitoyltransferase 1(CPT1)is a protein that catalyzes the rate-limiting step of FAO, controlling FAO directly(6). Among CPTI family, CPT1a is the most 
prevailing enzyme because of its wider distribution and better sensibility to their inhibitor malonyl-CoA(7). Targeting CPT1a has shown remarkable anti-leukemia activity: A novel CPT1a inhibitor ST1326 has been proved effective on leukemia cell lines and primary cells obtained from patients with hematologic malignancies(8). However, its prognostic value and possible treatment strategies are still unclear in AML.

A majority of leukemia cells have a survival advantage over normal cells because they fail to undergo apoptosis(9). Anti-apoptotic Bcl-2 protein is one such protein that is overexpressed in many cancers, which makes it an ideal target for cancer therapy(9). Venetoclax (ABT199) is an oral and highly selective bioavailable inhibitor targeting the $\mathrm{BH} 3$ domain of $\mathrm{Bcl}-2$ specifically(10). Compared to other drugs in its class, venetoclax has lower hematological toxicity(11). To date, it has been approved for the treatment of first-line and relapsed/refractory chronic lymphocytic leukemia (CLL) and AML(12). Unfortunately, despite its promising results in hematologic malignancies, intrinsic resistance is still a big problem(13). Previous study show that ABT199 (Venetoclax) has promising antileukaemic activity in AML therapy but increasing Mcl-1 limits its effect(14).

In this study, we found higher CPT1a levels are associated with poor prognosis and downregulation of it inhibits proliferation of AML cells, providing direct evidence for CPT1a as a prognostic biomarker for AML. The metabolic patterns identified its role of adverse prognostic biomarker. Moreover, our group combined ST1326 with ABT199 in AML cell lines and primary AML cells to identify the synergistic effect through downregulation of Mcl-1. We found ST1326 can inhibit pGSK3 $\beta$ and pERK to prevents upregulation of Mcl-1 induced by ABT199 effectively. Here, we provide a proper combinational therapy strategy to remedy the limited effect of CPT1a selective inhibitor, in the meanwhile, also solve the problem of resistence of ABT199.

\section{Materials And Methods}

Drugs

MG132 were purchased from Selleck Chemicals(Houston, Texas, USA). ST1326 and ABT199 was purchased from Sigma-Aldrich (St Louis, MO, USA)

Cell culture

HL-60, THP-1, OCl-AML2 and OCI-AML3 cell lines were purchased from Shanghai Cell Bank of the Chinese Academy of Sciences. KASUMI-1 cell line was gifted by Professor Chen Saijuan (Shanghai Institute of Hematology, Shanghai, China). These cells were cultured in RPMI 1640 medium supplemented with 10\% fetal bovine serum(FBS)(Gibco) at $37^{\circ} \mathrm{C}$ in a humidified incubator containing $5 \% \mathrm{CO} 2$. MV4-11 and MOLM-13 cell lines were a kind gift from Professor Ravi Bhatia (City of Hope National Medical Center, Duarte, CA, USA). These two cell lines were cultured in Iscove's Modified Dulbecco's Medium (IMDM) supplemented with $10 \%$ (FBS). 
Diagnostic AML patient samples were purified by standard Ficoll-Hypaque (Sigma-Aldrich) density centrifugation, then cultured in RPMI 1640 with 10\% FBS.

Clinical samples

Clinical data were collected from Zhejiang Institute of Hematology, China. Informed consent was provided from all patients according to institutional guidelines. This study was approved by the Ethics Committee of the First Affiliated Hospital of Zhejiang University. From July 2010 to July 2016, 325 patients were included in this study with detailed diagnostic and treatment information. Cytogenetically normal acute myeloid leukemia (CN-AML) was defined as AML with the karyotype $46 \mathrm{XY}$ [20] or $46 \mathrm{XX}$ [20] in all 20 metaphase cells analyzed. Gene mutations were analyzed by whole gene sequencing. Use descriptive statistics to summarize patient characteristics, including frequency counts, medians, and ranges.

Cell viability assay

AML Cells were seeded in $96-$ well plates at $1-2 \times 10^{4}$ (AML cell lines) or $1 \times 10^{5}$ (primary AML cells) per well. Then cells were treated with variable concentrations of ST1326 and/or ABT199 for 48 hours. Next, $10 \mu$ l MTS solution (Promega, Madison, WI) was added to each well. Cells were incubated for 4 hoursat $37^{\circ} \mathrm{C}$ in a humidified incubator containing $5 \%$ CO2.Finally, cells at plates were assessed at a wavelength of $490 \mathrm{~nm}$. For the AML cell lines, experiments were performed 3 independent times in triplicate, while primary patient sample experiments were performed once in triplicate due to limited sample.

RNA knockdown in human leukemia cell lines

To knock down CPT1a in human leukemia cell lines, short hairpin RNAs (shRNAs) were designed and cloned into amodified psi-LVRU6GP-shRNA plasmid. The sequences of sh1 was GCTCTTAGACAAATCTATCTC. The sequence of sh2 was GCCTTTGGTAAAGGAATCATC. The sequences of NC shRNA were ACAGAAGCGATTGTTGATC. These vectors were then packaged with human embryonic kidney 293T cells for the infection of leukemic cells. Then virus supernatant was collected at $48 \mathrm{~h}$ and $72 \mathrm{~h}$. AML cell lines were transfected with the shRNA or control lentiviruses and incubated for 72 h. Next, cells were continuously cultured in the medium containing $1.0 \mu \mathrm{g} / \mathrm{mL}$ puromycin.

Growth curve assay

Cells were seeded in 96-well plates $\left(1.0 \times 10^{4}\right.$ cells per well), blank medium as a control, $10 \mu \mathrm{L}$ of MTS solution (Promega CellTitre96) $(5 \mathrm{mg} / \mathrm{mL}$ ) were added to each well at $0 \mathrm{~h}, 24 \mathrm{~h}, 48 \mathrm{~h}$ and $72 \mathrm{~h}$, and the cells were incubated for an additional $4 \mathrm{~h}$ at $37^{\circ} \mathrm{C}$, the absorbance was measured at $490 \mathrm{~nm}$.

Annexin V/propidium iodide staining and flow cytometry analysis 
AML cells were treated with ST1326 or ABT199, alone or in combination for 48hours. Then cells were costainedwith Annexin VFITC and Propidium lodide (PI) for 15 min using an apoptosis detection kit (Beckman Coulter, Brea, CA, USA) in the dark. Apoptotic cells were analyzed by flow cytometry using FACScan ${ }^{\text {TM }}$ flow cytometer (Becton Dickinson, San Diego, CA, USA). Results are expressed as percent annexin $\mathrm{V}+$ cells.

Western blot analysis

Cells were lysed in radioimmunoprecipitation (RIPA) buffer (Cell Signaling Technology) on ice for $30 \mathrm{~min}$. After centrifugation of the cell lysate at $12000 \times \mathrm{g}$ for $15 \mathrm{~min}$ at $4^{\circ} \mathrm{C}$, Protein concentration of the cellular supernatant was determined using BCA reagent(BBI life science, Shanghai, China). Cell lysates were then loaded onto 10\% SDS-PAGE (Life Technologies, Carlsbad, CA, USA). After electrophoresis, proteins were transferred to PVDF membrane (Millipore, Billerica, MA, USA). Then, the membranes were blocked with $5 \%$ non-fat milk for $1 \mathrm{~h}$ and incubated withprimary antibodies overnight at $4^{\circ} \mathrm{C}$. Membranes were incubated with secondary antibodies (Cell Signaling Technology) for $1 \mathrm{~h}$ at room after washing three times with TBST buffer temperature. The target proteins were visualized using an ECL detection kit (Amersham, Little Chalfont, UK) and analyzed using Image Lab ${ }^{\text {TM }}$ software (Bio-Rad Laboratories, Hercules, CA, USA).

Primary antibodies for immunoblotting were purchased from the following sources: caspase3, cleaved caspase3, PARP, Bad, Bax, BCL-2, MCL-1, BCL-xl, BIM, p-ERK, ERK, p-AKT, AKT, pGSK3 $\beta$, GSK3 $\beta, p-$ Mcl1Thr163, GAPDH, $\beta$-tubulin and $\beta$-actin antibodies were purchased from Cell Signaling Technology (Beverly, MA,USA).

Real-time RT-PCR

Total RNA was isolated from the AML cells using TRIzol reagent according to the manufacturer's instructions. Reverse transcription was performed using the RNAPCR core kit (Life Technologies, Paisley, UK). Real-time quantitative PCR was performed on iQ5 System (Bio-Rad, Hercules, CA)using a SYBR Green qPCR master mix with GAPDH as an internal control. Primer sequences used are listed in following:

Mcl-1,5'-AAGAGGCTGGGATGGGTTTGTG-3'(forward),5'-TTGGTGGTGGTGGTGGTTGG-3'(reverse); CPT1a,5'-ATCAATCGGACTCTGGAAACGG-3'(forward), 5'-TCAGGGAGTAGCGCATGGT-3'(reverse); GAPDH,5'-GGAGCGAGATCCCTCCAAAAT-3'(forward) ,5'-GGCTGTTGTCATACTTCTCATGG-3'(reverse).

Sample Preparation and GC-TOFMS Analysis

12 bone marrow samples with aberrant CPT1a expression collected at disease diagnosis were stored frozen at $-80^{\circ} \mathrm{C}$ until use. Each $10^{7}$ of bone marrow blasts was added into a $1.5 \mathrm{~mL}$ of tube followed by the addition of $400 \mu \mathrm{L}$ of acetone for protein precipitation. The mixture was stirred by vortex for $30 \mathrm{~s}$ and centrifuged at $10000 \mathrm{rpm}$ for $10 \mathrm{~min}$. A 400- $\mu \mathrm{L}$ supernatant was transferred to a $500 \mu \mathrm{L}$ of glass tube and dried under vacuum. The dried analytes were dissolved in $80 \mu \mathrm{L}$ of methoxylamine hydrochloride (15 $\mathrm{mg} / \mathrm{mL}$, dissolved in pyridine) for $90 \mathrm{~min}$ at $30^{\circ} \mathrm{C}$ and then silylated with $80 \mu \mathrm{L} \mathrm{N}, \mathrm{O}$-bis-trimethylsilyl- 
trifluoroacetamideand Trimethylchlorosilane (in a ratio of 99:1) (Supelco) for $2 \mathrm{~h}$ at $70^{\circ} \mathrm{C}$. Each $70-\mu \mathrm{L}$ aliquot of hexane was added to the derivatization bottles. After the sample was stirred for $1 \mathrm{~min}$ and kept at room temperature for an hour, $1-\mu \mathrm{L}$ aliquot of the solution was injected into a PerkinElmer gas chromatography coupled with a TurboMass-Autosystem XL mass spectrometer (PerkinElmer, Inc.) in the splitless mode. A DB-5MS capillary column coated with 5\% Diphenyl cross-linked $95 \%$ dimethylpolysiloxane (30 m × $250 \mu \mathrm{m}$ i.d., 0.25- $\mu \mathrm{m}$ film thickness; Agilent J\&W Scientific, Folsom, CA) was used for separation. Both the injection temperature and the interface temperature were set to $260^{\circ} \mathrm{C}$, and the ion source temperature was adjusted to $200^{\circ} \mathrm{C}$. Initial GC oven temperature was set at $80^{\circ} \mathrm{C}$ for 2 min after injection, and was raised up to $285^{\circ} \mathrm{C}$ with $5^{\circ} \mathrm{C} / \mathrm{min}$ and maintained at $285^{\circ} \mathrm{C}$ for $7 \mathrm{~min}$. Helium at a flow rate of $1 \mathrm{~mL} / \mathrm{min}$ was used as the carrier gas. The measurements were made with electron impact ionization $(70 \mathrm{eV})$ in the full scan mode $(\mathrm{m} / \mathrm{z} 30-550)$. A total of 71 metabolites were identified by the comparison with the internal library built with the standard reference compounds.

\section{Statistical analysis}

AML patient characteristics were summarized using descriptive statistics, which included frequency counts, median and interquartile range. Categorical variables were compared using Fisher's exact test, and continuous variables were analyzed using a nonparameter T-test. OS was defined as time from the date of diagnosis until death due to any cause or the last follow-up. Univariate and multivariate analyses with a Cox proportional hazards models were performed to assess significant predictors. The proportional-hazards assumption was checked for each variable before fitting Cox models. Data were analyzed using GraphPad Prism 8.0 software. CalcuSyn software (Biosoft, Cambridge, UK) was used to calculate the combination index $(\mathrm{Cl})$.

\section{Results}

\section{Overexpression of CPT1a predicts poor clinical outcome in Chinese AML patients.}

First, we measured CPT1a mRNA expression of 325 AML patients and 8 normal people by real-time PCR. CPT1a mRNA expression was similar between primary AML samples $(n=325)$ and normal BM cells from healthy donors $(n=8)($ Fig. $1 A)$. According to the mRNA expression level of CPT1a, patients were classified into high expression group $(n=245,75 \%)$ and low expression group $(n=80,25 \%)$. We found that there was no statistical correlation between CPT1a expression and variables of gender, age, BM blasts, WBC levels, hemoglobin levels, platelet counts, FAB classifications and genes mutations(Table 1). In survival analyses, high CPT1a expression $(n=245)$ had a relatively short overall survival (OS) $(P=0.01$, log-rank test) and event free survival (EFS) $(P=0.032$, log-rank test) compared to patients in low expression group $(n=80)$ (Fig. 1B and C). And in the multivariable analysis, high CPT1a expression is associated with poor survival after adjusting age, WBC, ENL classification, DMNT3a and IDH1, IDH2 mutations regardless of OS [HR (95\% Cl), 1.674(1.097,2.557); $\mathrm{P}=0.017$, Table 2] or EFS [HR $(95 \% \mathrm{Cl})$, 1.412(0.965,2.066); $P=0.076$ Table S1]. To further investigate the biological role of CPT1a in AML, we classify 12 patients(Table S2) into high expression group $(n=6,50 \%)$ and low expression group $(n=6$, 
$50 \%$ ) according to the mRNA expression level of CPT1a and then performed a metabolomic analysis(Fig. 1D). As expected, we observed a decrease of fatty acid like tetracosanoic acid in CPT1a high expression group comparing to low expression group, which confirms that CPT1a can facilitate FAO to provide energy fueling tumor growth. Additionally, amino acid levels and urea cycle intermediates were decreased accompanied by increases in nucleotide synthesis, suggesting the utilization of more glucose and amino acids for the purpose of nucleotide synthesis and cellular proliferation. 
Table 1

Characteristics of CN-AML patients by high and low CPT1a expression

CPT1a expression

P-value

LOW expression $(\mathrm{N}=80) \quad$ High expression

$(\mathrm{N}=245)$

Number(\%)

Male,n(\%)

Age,median(IQR),years

BM blast, median(IQR), ${ }^{1}$

WBC, median (IQR), $₫ 10^{9} / \mathrm{L}^{2}$

$H B$, median(IQR),g/L ${ }^{3}$

$\mathrm{PLT}$, median $(\mathrm{IQR}), \mathbb{8} 10^{9} / \mathrm{L}^{4}$

FAB classification, $\mathrm{n}(\%)^{5}$
$8(10.0)$

7 ( 8.8)

39 (48.8)

1 (1.2)

3 ( 3.8$)$

$20(25.0)$

2 (2.5)

M6

Genes mutations,n(\%)

FLT3ITD

CEBPA $^{\text {DM6 }}$

NPM1
11(14.1)

10 (13.9)

$19(25.0)$
$245(75 \%)$

137 (56.6)

0.13

$56.00(39.75,65.00)$

0.054

$68.00(43.00,81.00)$

0.179

$10.50(2.38,45.98)$

0.336

$84.00(67.75,102.25)$

0.360

$49.00(26.00,94.75)$

0.199

$22(9.0)$

19 ( 7.8)

$122(49.8)$

$3(1.2)$

10 (4.1)

63 (25.7)

4 ( 1.6)

Abbreviations:1WBC, white blood cell; 2HB, hemoglobin; 3PLT, platelet counts; 4BM, bone marrow; 5FAB, French-American-British classification systems; 6DM: Double-allele. 7The protocols used for induction therapy in different groups including HAA, homoharringtonine-based treatment (homoharringtonine $2 \mathrm{mg} / \mathrm{m} 2 /$ day for 3 days, cytarabine $75 \mathrm{mg} / \mathrm{m} 2$ twice daily for 7 days, aclarubicin $12 \mathrm{mg} / \mathrm{m} 2$ daily for 7 days) regiment; DA, daunorubicin $45 \mathrm{mg} / \mathrm{m} 2$ daily for 3 days and cytarabine $100 \mathrm{mg} / \mathrm{m} 2$ daily for 7 days; IA, idarubicin 6-8 mg/m2 daily for 7 days and aclarubicin $20 \mathrm{mg} / \mathrm{m} 2$ daily for 5 days. IQR, interquantile. BMT, bone marrow transplantation. ELN (European leukemia Net) favorable genotype represents NPM1 mutant and FLT3-ITD negative or double allele CEBPA mutations 


\section{CPT1a expression}

P-value

\section{LOW expression $(\mathrm{N}=80) \quad$ High expression}

$(\mathrm{N}=245)$

DNMT3A

IDH1

IDH2
$5(7.1)$

$19(26.0)$

$7(10.8)$
$33(15.8)$

0.073

$42(20.3)$

0.325

$33(16.3)$

ELN favorable group,n(\%)

Treatment, $n(\%)^{7}$

CPT1A,median(IQR)

$0.58(0.30,0.78)$

$2.79(1.69,4.66)$

$<0.001$

Abbreviations:1WBC, white blood cell; 2HB, hemoglobin; 3PLT, platelet counts; 4BM, bone marrow; 5FAB, French-American-British classification systems; 6DM: Double-allele. 7The protocols used for induction therapy in different groups including HAA, homoharringtonine-based treatment (homoharringtonine 2 mg/m2/day for 3 days, cytarabine 75 mg/m2 twice daily for 7 days, aclarubicin $12 \mathrm{mg} / \mathrm{m} 2$ daily for 7 days) regiment; DA, daunorubicin $45 \mathrm{mg} / \mathrm{m} 2$ daily for 3 days and cytarabine $100 \mathrm{mg} / \mathrm{m} 2$ daily for 7 days; IA, idarubicin 6-8 mg/m2 daily for 7 days and aclarubicin $20 \mathrm{mg} / \mathrm{m} 2$ daily for 5 days. IQR, interquantile. BMT, bone marrow transplantation. ELN (European leukemia Net) favorable genotype represents NPM1 mutant and FLT3-ITD negative or double allele CEBPA mutations

Table 2

Univariate and multivariate overall survival analyses in CN-AML.

\begin{tabular}{|lllll|}
\hline & \multicolumn{2}{l}{ Univariate analysis } & \multicolumn{2}{l|}{ Multivariate analysis } \\
\hline Variables & P-value & $\mathrm{HR}(95 \% \mathrm{Cl})$ & P-value & $\mathrm{HR}(95 \% \mathrm{Cl})$ \\
\hline CPT1A expression & 0.005 & $1.693(1.167,2.454)$ & 0.017 & $1.674(1.097,2.557)$ \\
\hline age & $<0.001$ & $1.034(1.024,1.044)$ & $<0.001$ & $1.04(1.028,1.052)$ \\
\hline WBC & $<0.001$ & $1.004(1.002,1.006)$ & $<0.001$ & $1.006(1.003,1.008)$ \\
\hline ENL favorable group & $<0.001$ & $0.499(0.339,0.734)$ & $<0.001$ & $0.319(0.202,0.505)$ \\
\hline DNMT3a & 0.002 & $1.971(1.282,3.03)$ & 0.204 & $1.341(0.853,2.11)$ \\
\hline IDH1 & 0.084 & $1.386(0.957,2.007)$ & 0.037 & $1.626(1.03,2.567)$ \\
\hline IDH2 & 0.736 & $1.081(0.686,1.703)$ & 0.73 & $0.92(0.574,1.476)$ \\
\hline HR hazard ratio;Cl confidence interval & & & \\
\hline
\end{tabular}

\section{Downregulation of CPT1a inhibits proliferation of AML cells.}

We performed qPCR and Western blotting assay to examine the mRNA and protein levels of CPT1a in a panel of human AML cell lines. We found all leukemia cell lines constitutively expressed CPT1a and the expression of CPT1a in AML cell lines was different, among which THP-1 had the highest mRNA level 
and OCl-AML3 had the lowest mRNA level. Consistently, the expression level of CPT1a protein was similarly higher in THP-1, HL-60, KASUMI-1, AML-OCI2, and the lowest in OCl-AML3(Fig. 2A) We silenced the expression of CPT1a by introducing lentivirus-encoded shRNAs into THP-1 and HL-60 cell lines. ShRNAs can efficiently decrease the expression of CPT1a(Fig. 2B) and cause a significant reduction in proliferation of both THP-1 and HL-60 cell lines (Fig. 2C).

\section{Inhibition of CPT1a can Synergistically Enhance the Antileukemic Activity of ABT199}

Previous study demonstrates that CPT1 can interact with Bcl-2(15) and the truncated form of the proapoptotic Bcl-2 family member Bid (tBid) decreases CPT1 activity, which will be antagonized by Bcl-2 overexpression(16). So we assume that inhibiton of CPT1a have a combinational effect with ABT199. We silenced the expression of CPT1a and found the knockdown of CPT1a made THP-1 and HL-60 cell lines more sensitive to ABT199(Fig. 3A). A novel CPT1a inhibitor ST1326 has been proved effective on leukemia cell lines and primary cells obtained from patients with hematologic malignancies(8). In order to better connect to clinical application, THP-1, HL-60, Kasumi-1, MV4-11 and OCI-AML2 cell lines were used to evaluate the combinatorial effect of ST1326 and ABT199. The results showed that exposure to both ST1326 and ABT199 single treatment could inhibit AML cell proliferation in a dose-dependent manner, and what's more, co-administration of ST1326 and ABT199 resulted in a further increased inhibition of cell proliferation (Fig. 3B). Synergy(CI 1.0 ) was also observed in both ABT199-sensitive primary cells and ABT199-resisitent primary cells(Fig. 3C). The characteristics of the patient samples were presented in Table 3. The dose-effect curves were determined by CalcuSyn analyses (Fig. 3B and C, down panel). The $\mathrm{Cl}$ values were presented in Table 4. We demonstrated that ST1326 combined with ABT199 had a strong synergistic effect (CI区1.0) in AML cell lines and primary AML cells in vitro.

Table 3

Characteristics of primary AML patients.

\begin{tabular}{|lllllll|}
\hline & Diagnose & Gender & Age(year) & $\begin{array}{l}\text { FAB } \\
\text { type }\end{array}$ & Cytogenetics & Molecular \\
\hline AML\#1 & refractory & female & 30 & M2a & $46, X X$ & NARAS \\
AML\#2 & de novo & female & 40 & NA & $46, X X$ & BCR/ABL BCORL1 \\
AML\#3 & refractory & male & 63 & M2 & $46, X Y$ & KMT2C \\
AML\#4 & de novo & female & 64 & M2 & $46, X X$ & RUNX1 \\
AML\#5 & refractory & female & 67 & M4 & $46, X X$ & SH2B3 \\
AML\#6 & refractory & female & 33 & M5 & $46, X X$ & \\
AML\#7 & de novo & female & 33 & M4 & $46, X X$ & FLT3-ITD NPM1 \\
AML\#8 & refractory & female & 73 & M0 & $46, X X$ & WT1 MLL-AF9 \\
& & & & & & CBFB-MYH11 WT1 \\
\hline
\end{tabular}


Table 4

$-1$

\begin{tabular}{|llll|}
\hline \multicolumn{4}{|c|}{ Combination Index Values } \\
\hline AML cell llines & ED50 & ED75 & ED90 \\
\hline THP-1 & 0.26890 & 0.24955 & 0.23277 \\
\hline Kasumi-1 & 0.25727 & 0.32034 & 0.87657 \\
\hline MV4-11 & 0.84190 & 0.51618 & 0.39560 \\
\hline OCI-AML2 & 0.63777 & 0.23040 & 0.27239 \\
\hline HL-60 & 0.48821 & 0.32436 & 0.21859 \\
\hline
\end{tabular}

Table 4

$-2$

\begin{tabular}{|llll|}
\hline \multicolumn{4}{|c|}{ Combination Index Values } \\
\hline Patient & ED50 & ED75 & ED90 \\
\hline AML\#1 & 0.67927 & 0.36933 & 0.23918 \\
\hline AML\#2 & 0.94233 & 0.60820 & 0.55849 \\
\hline AML\#3 & 1.39321 & 0.18723 & 0.18043 \\
\hline AML\#4 & 2.26997 & 0.68618 & 0.29174 \\
\hline AML\#5 & 0.18060 & 0.02656 & 0.00498 \\
\hline AML\#6 & 0.88419 & 0.91904 & 0.96458 \\
\hline AML\#7 & 0.41894 & 0.41487 & 0.60241 \\
\hline AML\#8 & 0.33128 & 0.31352 & 0.35354 \\
\hline
\end{tabular}

Combination of ST1326 and ABT199 results in synergistic induction of apoptosis and ST1326 prevents up-regulation of Mcl-1 induced by ABT199

To explore the mechanism of synergistic effect, we treated THP-1 and HL-60 cells with ST1326 and ABT199 at low and high concentrations separately or synergistically for $24 \mathrm{~h}$ and then measured cell death by Annexin V/DAPI dual staining. Compared with single agents, combination of ST1326 and ABT199 resulted in a significant increase in apoptosis (Fig. 4A). Moreover, expression of cleaved caspase- 3 and cleaved PARP was higher in cells cultured with combinational agents compared with that observed for ST1326 and ABT199 (Fig. 4B). Next, we analyzed the key signaling molecules of relevant $\mathrm{Bcl}-2$ family proteins, which has great relevant with apoptosis pathway. The levels of Bcl-2, Bax, Bad, Bim and Bcl-xl remained relatively unchanged (Fig. 4C). ST1326 treatment decreased Mcl-1 levels and prevented up-regulation of Mcl-1 induced by ABT199(Fig. 4C). Mcl-1 was further up-regulated after shortterm exposure to ABT199(Fig. 4D), which further enhance their resistance to ABT199. 


\section{ST1326 inhibits PGSK3 $\beta$ and pERK to downregulate Mcl-1.}

To find how ST1326 treatment reduces Mcl-1 protein level, we first performed RT-PCR. Interestingly, Mcl-1 transcript levels were not decreased in THP-1 and HL-60 cells treated with ST1326 and ABT199, alone or combined for 24 hour, indicating a post-transcriptional mechanism(Fig. 5A). Then, a proteasome inhibitor MG132 was used to examine Mcl-1 protein stability. THP-1 and HL-60 cells were treated with ST1326 or MG132 alone or in combination. MG132 pretreatment could suppress ST1326-induced MCL1 downregulation in THP-1 cells and HL-60 cells(Fig. 5B). These results indicate that the reduction of the Mcl-1 protein levels in AML cells treated with ST1326 might be mediated through proteasome degradation. Previous studies have shown that AKT-mediated GSK3 $\beta$ phosphorylation and MAPK-mediated Mcl-1 phosphorylation are involved in Mcl-1 degradation $(17,18,19,20,21,22,23)$. Therefore, we evaluated the effect of ST1326 on MAPK and AKT activation. ST1326 treatment decreased the phosphorylation of GSK3 $\beta$ at Ser9 and p-AKT(Fig. 5C) and decreased pERK along with pMcl-1Thr163 (Fig. 5D). To further examine the role ERK played in Thr163 phosphorylation, we tested whether ERK inhibition would lead to reduced levels of pMcl-1Thr163. Consistently, inactivation of ERK by PD98059 (ERK/MAPK inhibitor) decreased Mcl-1 expression and its phosphorylation at Thr163(Fig. 5E).

\section{Discussion}

Warburg effect proposed the idea that cancer cells provide energy through increased glycolysis in the presence of oxygen because of respiratory defect(24). In addition to changes in glucose metabolism, there is convincing evidence that cancer cells have specific changes in lipid metabolism. Fatty acid oxidation (FAO) is a way to produce adenosine triphosphate, reduced nicotinamide adenine dinucleotide phosphateand acetyl-coenzyme A in cancer cells(5). As an enzyme of the rate-limiting step of FAO, carnitine palmitoyl transferase1a(CPT1a) plays an important role in cancer metabolic adaptation. Previous study found overexpression of CPT1a was shown in AML than normal BM and PB and high expression of CPT1a is associated with adverse outcomes in AML using public microarray datasets with bioinformatics method(25). In this study, we validated that opinion CPT1a is a high risk prognostic factor for AML again with our data of 325 Chinese patients. In parallel, downregulated expression of CPT1a using shRNAs inhibits proliferation of AML cells. However, we found CPT1a mRNA expression was similar between primary AML samples $(n=325)$ and healthy donors $(n=8)$. What's more, we explore the distinctive metabolic patterns associated with CPT1a expression in AML. In high expression of CPT1a group, the decreased expression of the fatty acid, amino acid levels and increasing expression of nucleotide synthesis imply CPT1a expression acts on an oncogene by facilitation of FAO and the utilization of more glucose and amino acids.

Previous study demonstrates that CPT1 can interact with Bcl-2(14) and the truncated form of the proapoptotic Bcl-2 family member Bid (tBid) decreases CPT1 activity, which will be antagonized by Bcl-2 over-expression(15). Taken together, we hypothesized that down-regulation of CPT1a sensitizes ABT199 to AML cells. The results verified our thoughts. What' more, we discovered for the first time that coadministration of ST1326 and ABT199 resulted in a further increased inhibition of proliferation in AML 
cell lines and primary patients. Combination of ST1326 and ABT199 results in synergistic induction of apoptosis.

Previous study demonstrated that the IC50 of venetoclax was inversely correlated with Bcl-2/Mcl-1 transcript ratio, and over-expression of $\mathrm{Bcl}-\mathrm{xl}$ or $\mathrm{Mcl}-1$ conferred resistance to venetoclax-induced apoptosis in AML cell lines(26). In our study, we observed that ST1326 could prevent up-regulation of Mcl-1 induced by ABT199 through proteasome degradation. Previous studies have shown that AKTmediated GSK3 $\beta$ phosphorylation and MAPK-mediated MCL1 phosphorylation are involved in MCL1 degradation $(17,18,19,20,21,22,23)$. In our study, ST1326 treatment decreased the phosphorylation of GSK3 $\beta$ at Ser9 and p-AKT and decreased pERK along with pMcl-1Thr163.

Preveious study show AML is rarely cured by a single enzyme or pathway, so it is likely that drugs targeting CPT1a will need to be combined with chemotherapy or other targeted drugs to succeed(5). Meanwhile, in the case of AML, venetoclax is best combined with another agent because resistance seems to develop rather quickly with venetoclax monotherapy(26). Our study may provide a proper scheme for this dilemma.

\section{Conclusion}

In conclusion, our study indicates that overexpression of CPT1a predicts poor clinical outcome in AML. CPT1a-selective inhibitor ST1326 combined with Bcl-2 inhibitor ABT199 showed strongsynergistic inhibitory effects on AML.

\section{Abbreviations}

Fatty acid oxidation (FAO)

Carnitine palmitoyltransferase $1 \mathrm{a}(\mathrm{CPT} 1 \mathrm{a})$

Acute myeloid leukemia (AML)

\section{Declarations}

Ethics approval and consent to participate: human data have been performed in accordance with the Declaration of Helsinki and have been approved by Clinical Research Ethics Committee of the First Affiliated Hospital, College of Medicine, Zhejiang University(IIT20200A).

Consent for publication: Not applicable

Availability of data and materials: The data that support the findings of this study are available from Key Laboratory of Hematologic Malignancies, Diagnosis and Treatment but restrictions apply to the availability of these data, which were used under license for the current study, and so are not publicly 
available. Data are however available from the authors upon reasonable request and with permission of Key Laboratory of Hematologic Malignancies, Diagnosis and Treatment.

Competing interests:The authors declare that they have no conflict of interest.

Funding:This work is supported by Key international cooperation projects of the National Natural Science

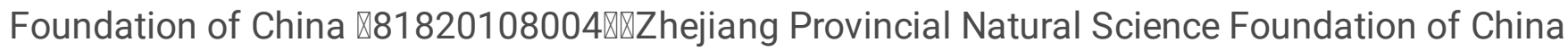
(LY19H080009), National Natural Science Foundation of China grants (81470305-H0812, U1404806), University Science and Technology Innovation Talent Support Program of Henan Province (17HASTIT046) and the Natural Science Foundation of Zhejiang Province (LY20H080008).

Authors' contributions:Sh M and QL performed the research. JJ and Jh W designed the research study. Ygi W, XH and Js H contributed essential reagents and tools. Jj P, FI L, Sj H, WI Y, Ww W, Xj L and Y Q collected clinical samples and analysed the data. Sh $\mathrm{M}$ and $\mathrm{Jh} \mathrm{W}$ wrote the paper.

Acknowledgements: Not applicable

\section{References}

1. Bonnet $D$, Dick JE. Human acute myeloid leukemia is organized as a hierarchy that originates from a primitive hematopoietic cell. Nature medicine. 1997;3(7):730-7.

2. Yates JW, Wallace HJ Jr, Ellison RR, Holland JF. Cytosine arabinoside (NSC-63878) and daunorubicin (NSC-83142) therapy in acute nonlymphocytic leukemia. Cancer chemotherapy reports. 1973;57(4):485-8.

3. Yates J, Glidewell O, Wiernik P, Cooper MR, Steinberg D, Dosik H, et al. Cytosine arabinoside with daunorubicin or adriamycin for therapy of acute myelocytic leukemia: a CALGB study. Blood. 1982;60(2):454-62.

4. Siegel RL, Miller KD, Jemal A. Cancer statistics. 2018. CA: a cancer journal for clinicians. 2018;68(1):7-30.

5. Carracedo A, Cantley LC, Pandolfı PP. Cancer metabolism: fatty acid oxidation in the limelight. Nature reviews Cancer. 2013;13(4):227-32.

6. Bonnefont JP, Djouadi F, Prip-Buus C, Gobin S, Munnich A, Bastin J. Carnitine palmitoyltransferases 1 and 2: biochemical, molecular and medical aspects. Mol Aspects Med. 2004;25(5-6):495-520.

7. Qu Q, Zeng F, Liu X, Wang QJ, Deng F. Fatty acid oxidation and carnitine palmitoyltransferase I: emerging therapeutic targets in cancer. Cell death disease. 2016;7(5):e2226.

8. Ricciardi MR, Mirabilii S, Allegretti M, Licchetta R, Calarco A, Torrisi MR, et al. Targeting the leukemia cell metabolism by the CPT1a inhibition: functional preclinical effects in leukemias. Blood. 2015;126(16):1925-9.

9. Radha G, Raghavan SC. Bcl-2: A promising cancer therapeutic target. Biochimica et biophysica acta Reviews on cancer. 2017;1868(1):309-14. 
10. Souers AJ, Leverson JD, Boghaert ER, Ackler SL, Catron ND, Chen J, et al. ABT-199, a potent and selective BCL-2 inhibitor, achieves antitumor activity while sparing platelets. Nature medicine. $2013 ; 19(2): 202-8$.

11. Juárez-Salcedo LM, Desai V, Dalia S. Venetoclax: evidence to date and clinical potential. Drugs in context. 2019;8:212574.

12. Deeks ED. Venetoclax. First Global Approval Drugs. 2016;76(9):979-87.

13. Campos EDV, Pinto R. Targeted therapy with a selective BCL-2 inhibitor in older patients with acute myeloid leukemia. Hematology transfusion cell therapy. 2019;41(2):169-77.

14. Niu X, Zhao J, Ma J, Xie C, Edwards H, Wang G, et al. Binding of Released Bim to Mcl-1 is a Mechanism of Intrinsic Resistance to ABT-199 which can be Overcome by Combination with Daunorubicin or Cytarabine in AML Cells. Clinical cancer research: an official journal of the American Association for Cancer Research. 2016;22(17):4440-51.

15. Giordano A, Calvani M, Petillo O, Grippo P, Tuccillo F, Melone MA, et al. tBid induces alterations of mitochondrial fatty acid oxidation flux by malonyl-CoA-independent inhibition of carnitine palmitoyltransferase-1. Cell death differentiation. 2005;12(6):603-13.

16. Paumen MB, Ishida $Y$, Han $H$, Muramatsu $M$, Eguchi $Y$, Tsujimoto $Y$, et al. Direct interaction of the mitochondrial membrane protein carnitine palmitoyltransferase I with Bcl-2. Biochem Biophys Res Commun. 1997;231(3):523-5.

17. Opferman JT. Unraveling MCL-1 degradation. Cell death differentiation. 2006;13(8):1260-2.

18. Maurer U, Charvet C, Wagman AS, Dejardin E, Green DR. Glycogen synthase kinase-3 regulates mitochondrial outer membrane permeabilization and apoptosis by destabilization of MCL-1. Molecular cell. 2006;21(6):749-60.

19. Cohen P, Frame S. The renaissance of GSK3. Nature reviews Molecular cell biology. 2001;2(10):76976.

20. Niu X, Wang G, Wang Y, Caldwell JT, Edwards H, Xie C, et al. Acute myeloid leukemia cells harboring MLL fusion genes or with the acute promyelocytic leukemia phenotype are sensitive to the Bcl-2selective inhibitor ABT-199. Leukemia. 2014;28(7):1557-60.

21. Ding Q, He X, Hsu JM, Xia W, Chen CT, Li LY, et al. Degradation of Mcl-1 by beta-TrCP mediates glycogen synthase kinase 3-induced tumor suppression and chemosensitization. Molecular cellular biology. 2007;27(11):4006-17.

22. Nifoussi SK, Vrana JA, Domina AM, De Biasio A, Gui J, Gregory MA, et al. Thr 163 phosphorylation causes Mcl-1 stabilization when degradation is independent of the adjacent GSK3-targeted phosphodegron, promoting drug resistance in cancer. PloS one. 2012;7(10):e47060.

23. Domina AM, Vrana JA, Gregory MA, Hann SR, Craig RW. MCL1 is phosphorylated in the PEST region and stabilized upon ERK activation in viable cells, and at additional sites with cytotoxic okadaic acid or taxol. Oncogene. 2004;23(31):5301-15.

24. Warburg O. On the origin of cancer cells. Science. 1956;123(3191):309-14. 
25. Shi J, Fu H, Jia Z, He K, Fu L, Wang W. High Expression of CPT1a Predicts Adverse Outcomes: A Potential Therapeutic Target for Acute Myeloid Leukemia. EBioMedicine. 2016;14:55-64.

26. Pullarkat VA, Newman EM. Bcl-2 Inhibition by Venetoclax: Targeting the Achilles' Heel of the Acute Myeloid Leukemia Stem Cell? Cancer discovery. 2016;6(10):1082-3.

\section{Figures}

A

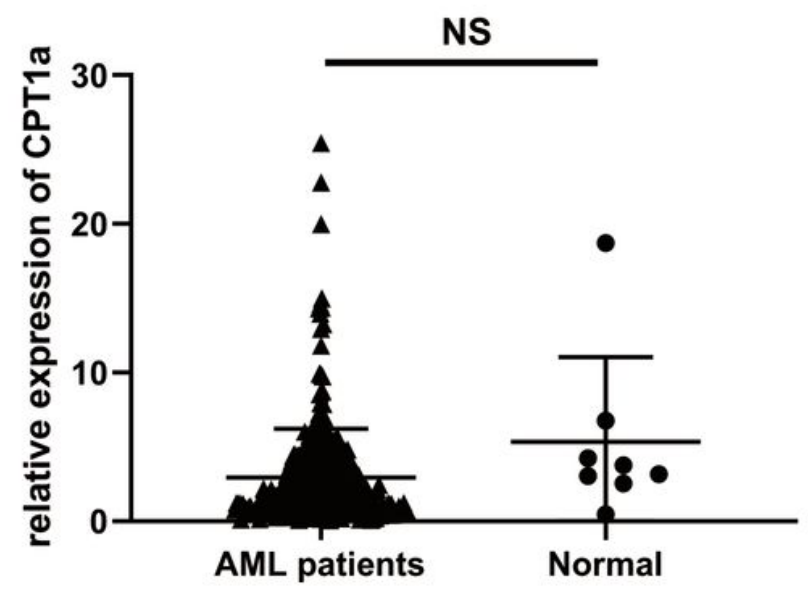

B

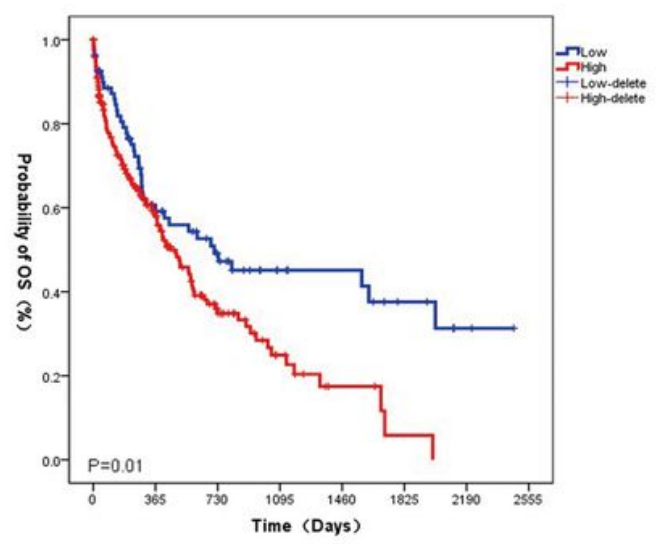

C

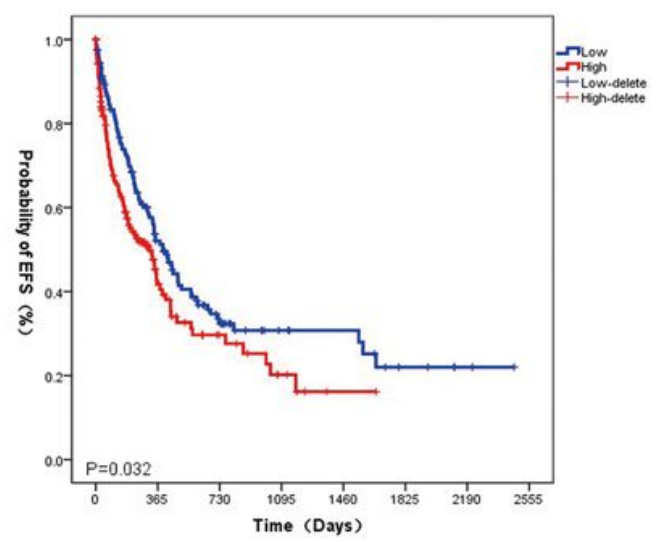

D $\quad \underset{\substack{0.4 \\ \text { - color Key }}}{\substack{0 \\ \text { value }}}$

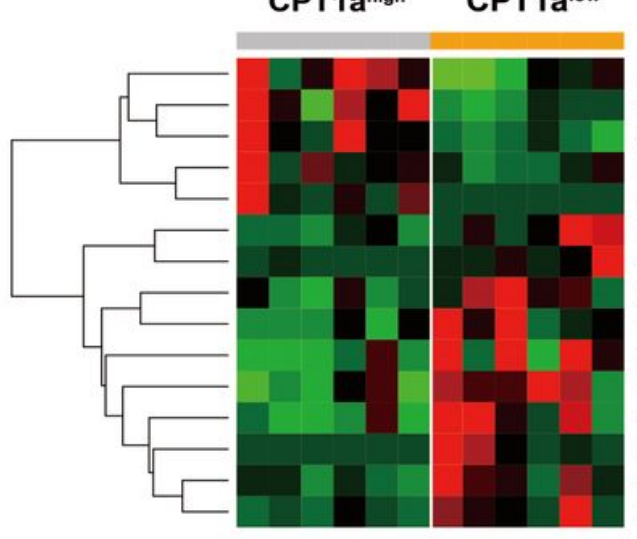

Ratio of Guanine/Guanosine

Guanine

Adenine

Ratio of Hypoxanthine/Inosine

Ratio of D-Fructose/Sucrose

Petroselinic acid

Sucrose

Sorbitol

Gluconic acid

L-Cystine

Urea

Tetracosanoic acid

D-Maltose

4-Hydroxyphenylpyruvic acid

Ratio of 4-Hydroxyphenylpyruvic acid/L-Tyrosine

Figure 1

(A) qRT-PCR analysis of CPT1a mRNA expression (mean \pm SEM)in normal BM cells $(n=8)$ and CN-AML samples $(n=325)$ (t-test). (B)Kaplan-Meier analysis of overall survival(OS) according to CPT1a mRNA 
expression in primary blasts from $325 \mathrm{AML}$ patients. (C) Kaplan-Meier analysis of event free survival (EFS) according to CPT1a mRNA expression in primary blasts from 325 AML patients. (D)Global metabolomics profile between high and low expression of CPT1a of 12 patients.

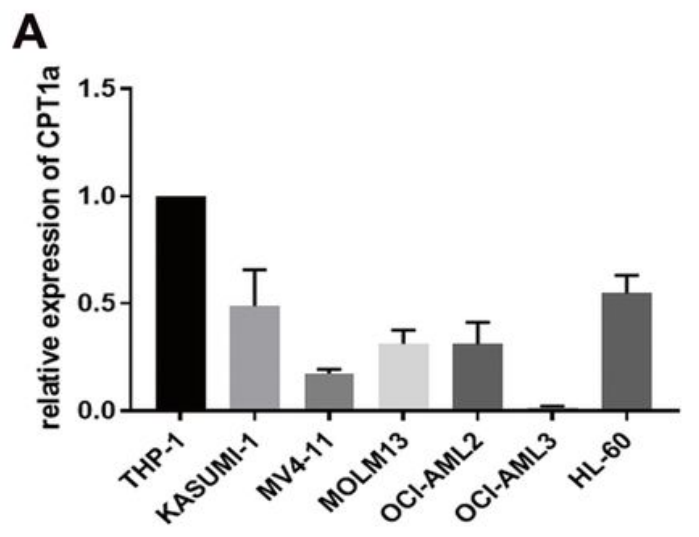

THP-1 Kasumi-1 MV4-11 Molm13 OCI-AML2 OCI-AML3 HL-60

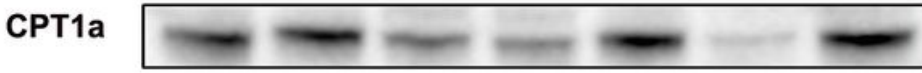

GAPDH

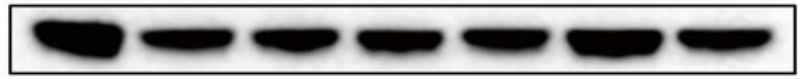

THP-1

C

B

THP-1

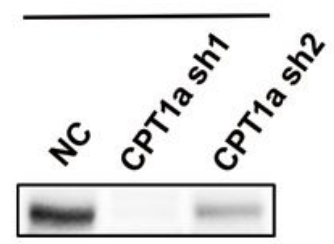

GAPDH

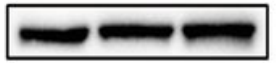

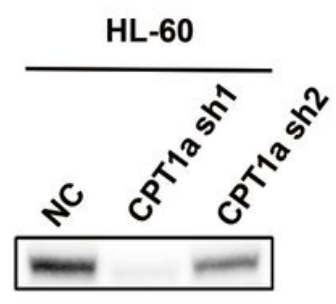

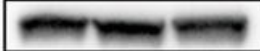

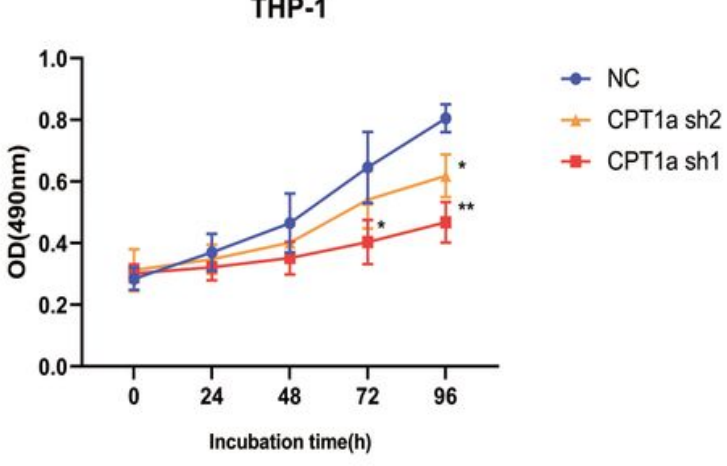

HL-60

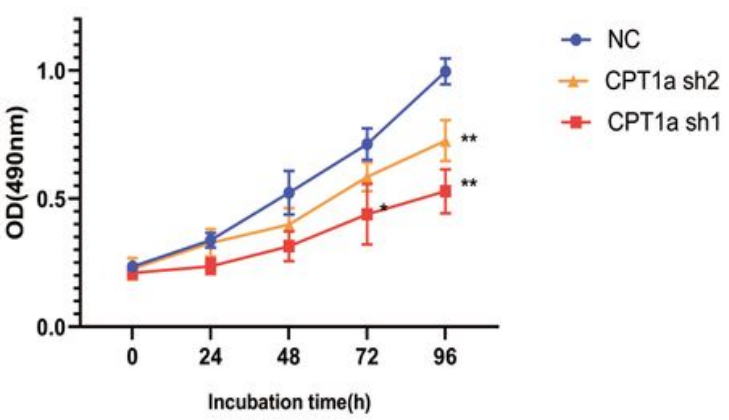

Figure 2

(A)qPCR and Western blotting analysis of CPT1a expression in human AML cell lines. (B)Western blotting analysis of the CPT1a protein level in THP-1 and HL-60 cells transduced with 2 different CPT1a shRNAs. The NC shRNA was used as a knockdown control. (C)Cell viability at 0h, 24h, 48h and 72h in THP-1 and HL-60 cells transduced with 2 different CPT1a shRNAs. 
A
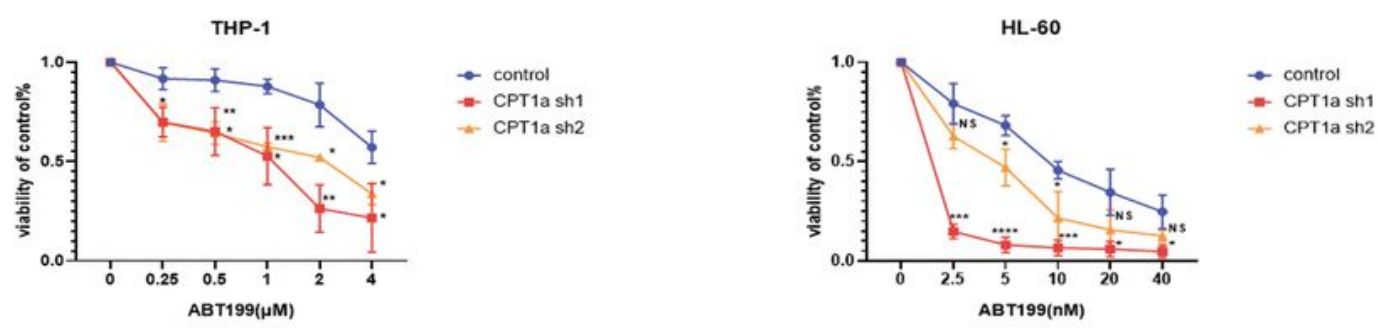

B
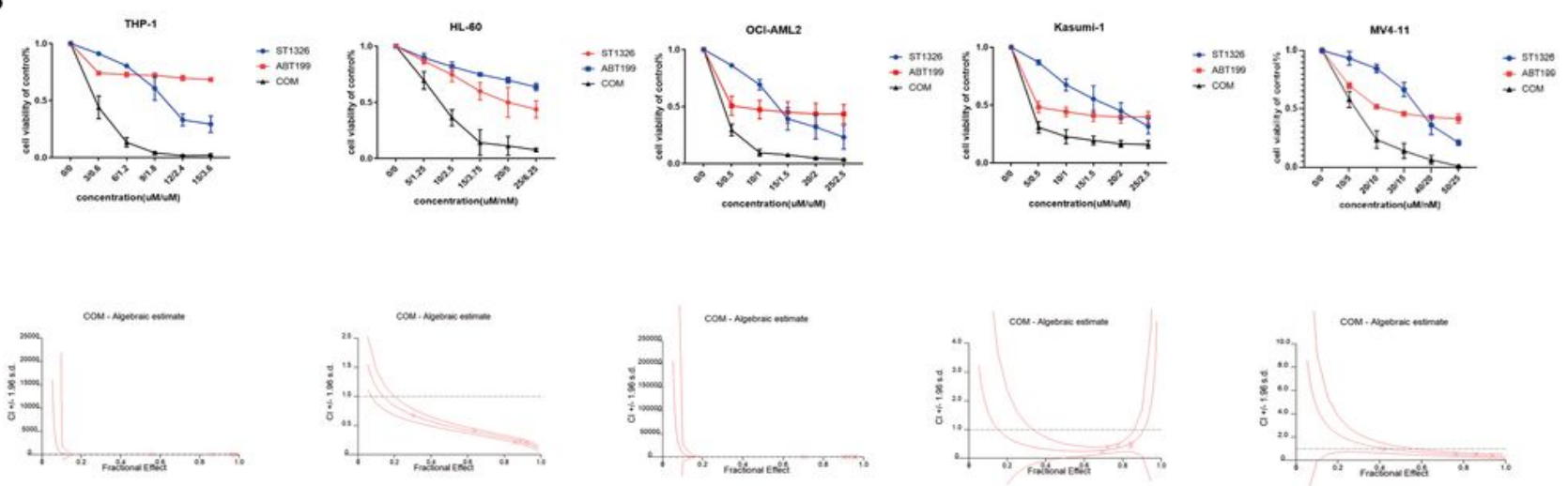

$\mathbf{C}$
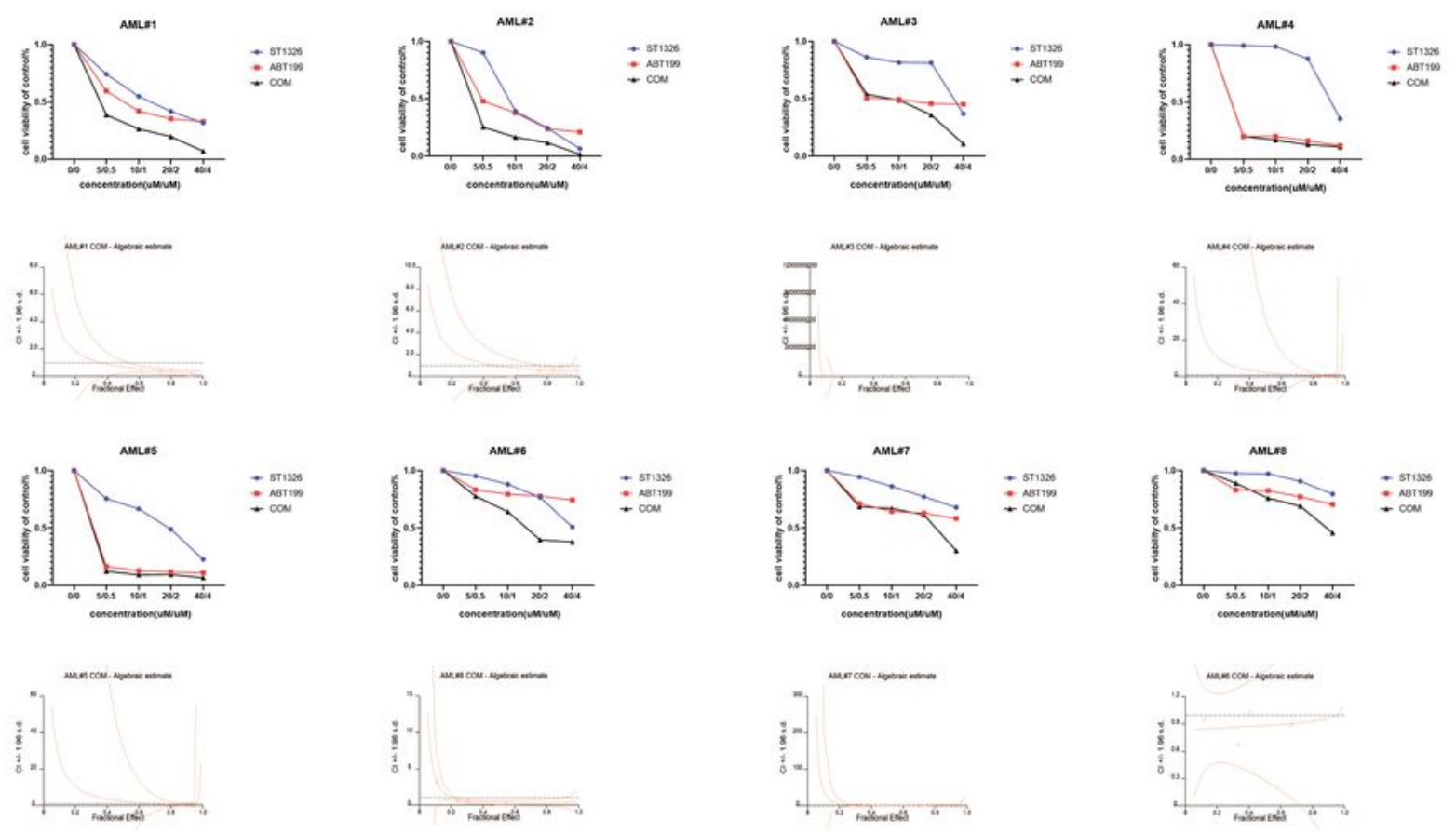

\section{Figure 3}

$(A, B)$ Cell viability after treatment with ST1326, ABT199 or combination in AML cell lines (A, up panel) and primary $A M L$ cells ( $B$, up panel) measured by MTS assay. The combination index $(C I)$ ( $A$ and $B$, down panel) was calculated using CalcuSyn software. The data are presented as mean \pm SD fromat least three independent experiments for cell lines. For patient sample, the MTS assay was performed once due to limited sample. 
A

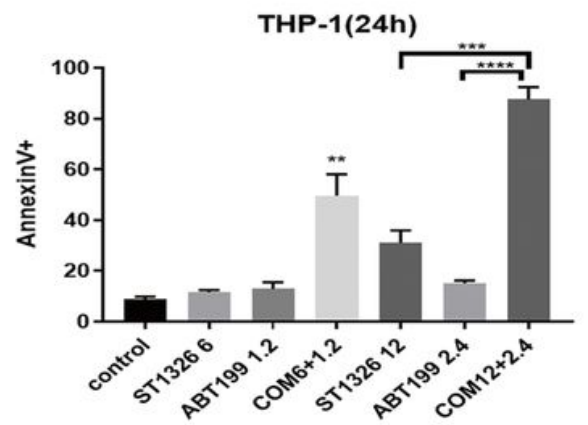

HL-60 (24h)

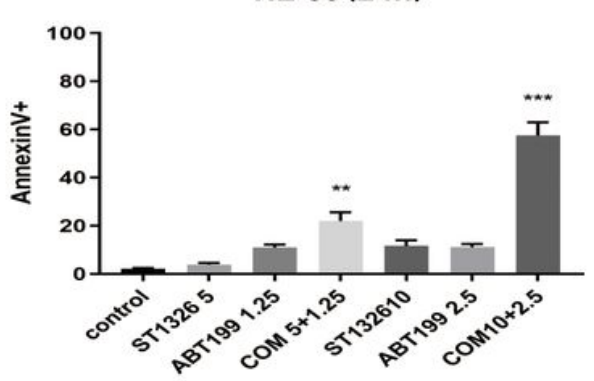

C

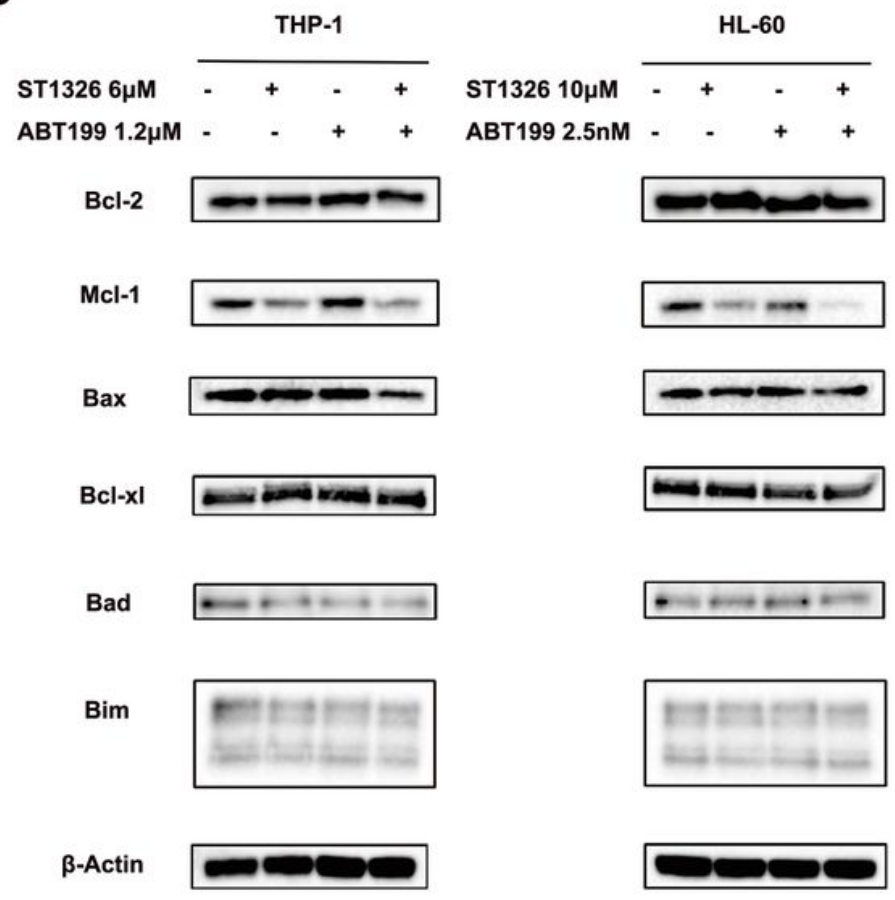

B

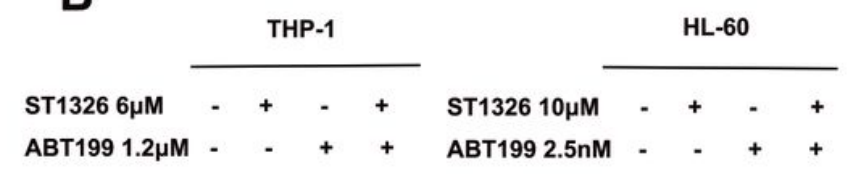

PARP
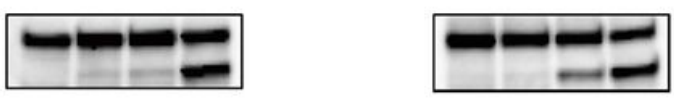

Caspase3
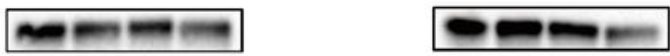

cf-Caspase 3
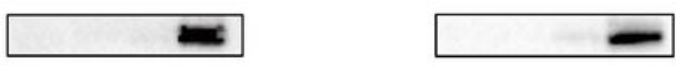

GAPDH
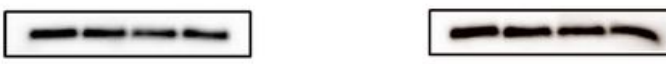

D

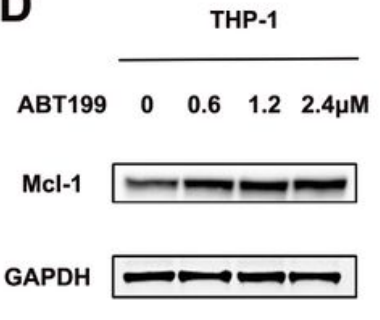

HL-60 $\begin{array}{lllll}\text { ABT199 } & 0 & 1.25 & 2.5 & 5 n M\end{array}$

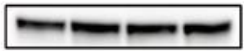

GAPDH

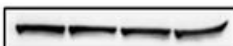

\section{Figure 4}

(A) Apoptosis induced by various treatments at $24 \mathrm{~h}\left({ }^{* *} \mathrm{P} \otimes 0.01\right.$, ${ }^{\star \star \star} \mathrm{P}<0.001 \rrbracket \star \star \star \star \mathrm{P}<0.0001$, unpaired $\mathrm{t}$ test, combination treatments versus single treatments). (B)THP-1, HL-60 cells were treated with ST1326 and ABT199, alone or combined, for $24 \mathrm{~h}$. Western blot of Caspase-3, cleaved Caspase-3 and PARP-1 in AML cells. GAPDH served as a loading control. (C) THP-1 and HL-60 cells were treated with ST1326 and ABT199, alone or combined, for $24 \mathrm{~h}$. Western blot of Bcl-2, Bax, Bad, Bcl-xL, Mcl-1 and Bim in AML cells. 
$\beta$-Actin served as a loading control. $\triangle \mathrm{D} \otimes \mathrm{THP}-1$ and HL-60 cells were treated with increasing doses of ABT199 for $24 \mathrm{~h}$. Western blot analysis was conducted for Mcl-1 protein levels.

A

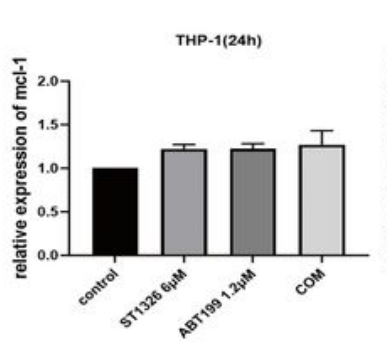

C

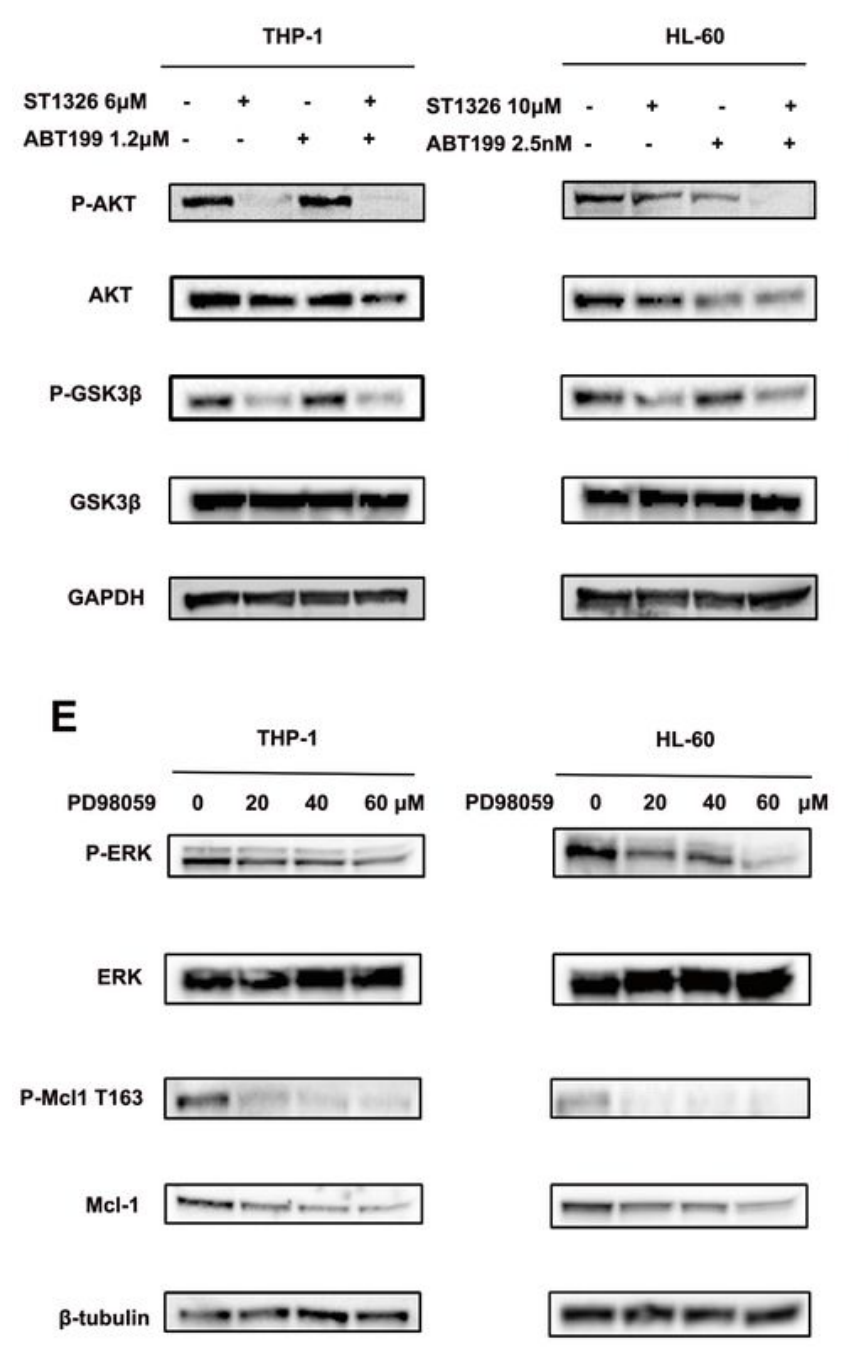

B
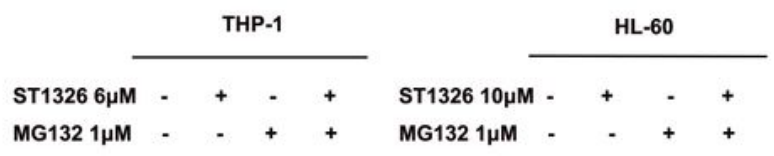

Mcl-1

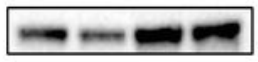

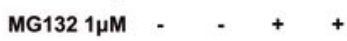

GAPDH

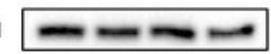

D

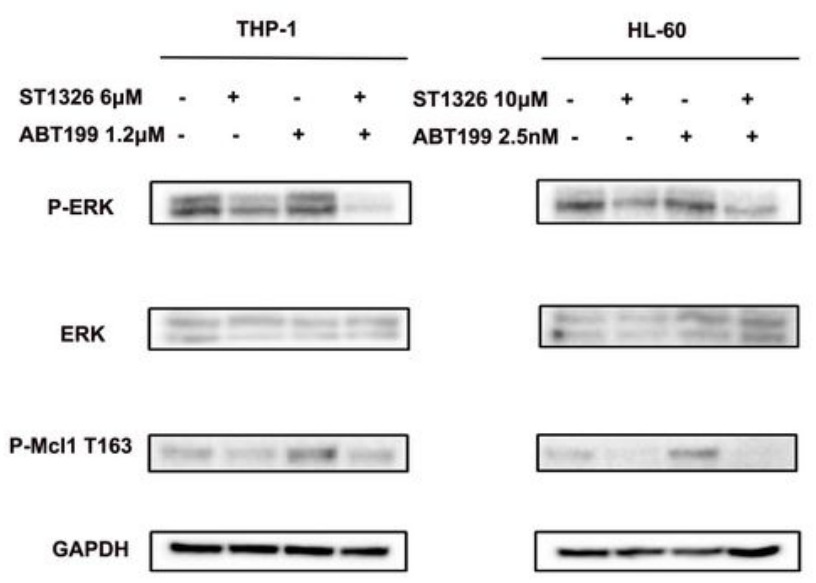

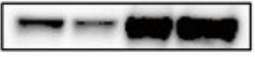

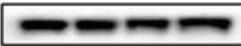


1 protein levels. (C) THP-1 and HL-60 cells were treated with ST1326 and ABT199, alone or combined, for $24 \mathrm{~h}$. Western blot analysis was conducted for p-GSK3 $\beta$, GSK3 $\beta$, AKT, p-AKT protein levels (D) THP-1 and HL-60 cells were treated with ST1326 and ABT199, alone or combined, for $24 \mathrm{~h}$. Western blot analysis was conducted for $\mathrm{p}-\mathrm{ERK}$, ERK, p-Mcl1 Thr163 protein levels. (E)THP-1 and HL-60 cells were treated with increasing doses of PD 98059 for 24 h.Western blot analysis was conducted for p-ERK, ERK, p-Mcl1 Thr163,Mcl-1 protein levels. 